\title{
Achievement of dietary fatty acid intakes in long-term controlled intervention studies: approach and methodology
}

\author{
MC Nydahl' ${ }^{1}$, RD Smith ${ }^{1}$, CNM Kelly ${ }^{1}$, BA Fielding ${ }^{2}$ and CM Williams ${ }^{1, *}$ \\ ${ }^{1}$ Hugh Sinclair Unit of Human Nutrition, Department of Food Biosciences, University of Reading, Reading RG6 6AP, \\ UK: ${ }^{2}$ Oxford Lipid Metabolism Group, Nuffield Department of Clinical Medicine, University of Oxford, \\ Oxford OX2 6HE, UK
}

Submitted 6 September 2000: Accepted 26 July 2001

\begin{abstract}
Objective: To describe the calculations and approaches used to design experimental diets of differing saturated fatty acid (SFA) and monounsaturated fatty acid (MUFA) compositions for use in a long-term dietary intervention study, and to evaluate the degree to which the dietary targets were met.

Design, setting and subjects: Fifty-one students living in a university hall of residence consumed a reference (SFA) diet for 8 weeks followed by either a moderate MUFA (MM) diet or a high MUFA (HM) diet for 16 weeks. The three diets were designed to differ only in their proportions of SFA and MUFA, while keeping total fat, polyunsaturated fatty acids (PUFA), trans-fatty acids, and the ratio of palmitic to stearic acid, and $n-6$ to $n-3$ PUFA, unchanged.

Results: Using habitual diet records and a standardised database for food fatty acid compositions, a sequential process of theoretical fat substitutions enabled suitable fat sources for use in the three diets to be identified, and experimental margarines for baking, spreading and the manufacture of snack foods to be designed. The dietary intervention was largely successful in achieving the fatty acid targets of the three diets, although unintended differences between the original target and the analysed fatty acid composition of the experimental margarines resulted in a lower than anticipated MUFA intake on the HM diet, and a lower ratio of palmitic to stearic acid compared with the reference or MM diet.

Conclusions: This study has revealed important theoretical considerations that should be taken into account when designing diets of specific fatty acid composition, as well as practical issues of implementation.
\end{abstract}

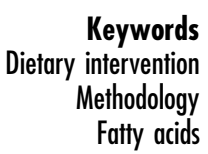

Current dietary recommendations aimed at reducing the incidence of coronary heart disease emphasise the need for reductions in total fat and saturated fatty acids (SFA) $)^{1,2}$. However, specific recommendations for changes in intakes of monounsaturated (MUFA) and polyunsaturated (PUFA) fatty acids have been more difficult to define due to insufficient and inconsistent evidence about their potential effects. Lack of knowledge in this area has driven the need for well-controlled intervention studies to investigate further the effects of individual fatty acids in human subjects and strengthen the scientific basis for making population dietary recommendations.

There has been considerable interest in recent years in the effects of different dietary fatty acids on plasma lipids $^{3-6}$ and other non-lipid cardiovascular risk factors, including low-density lipoprotein oxidisability, haemostatic factors and immune function ${ }^{7-10}$. Great emphasis is placed on the validity of the biomarkers used in such studies. However, very few studies provide detailed information on the methods used to achieve experimental target fatty acid intakes. It is now evident that there are a number of important design criteria that need to be considered when planning dietary intervention studies involving controlled changes in dietary fatty acids intake ${ }^{11}$.

Whilst small changes in fatty acid intake may be achieved by the use of supplement capsules, more extensive changes require modifications to the diet as a whole. Consideration needs to be given to the design of the experimental diets, to enable fatty acid targets to be achieved whilst maintaining constant intakes of other nutrients, and providing subjects with a diet that is palatable, practical to implement, nutritionally adequate and sufficiently varied to maintain compliance throughout the entire study period. Short-term studies carried out under metabolic-ward conditions have the advantage of being able to control energy and nutrient intakes strictly, with all food being prepared for the subjects $^{12-14}$. However, it is not usually feasible to assess 
the longer-term effects of dietary changes on healthy individuals under such conditions. Most dietary intervention studies of several weeks or months have been carried out in free-living subjects who are provided with intervention foods and/or instructed how to modify their diet, while continuing with their normal lifestyle $e^{15-17}$. Under these circumstances, it is particularly important that the diet is acceptable to the subjects and practical to implement, and regular monitoring by the investigators is required to ensure that the diet is well controlled and that compliance is maintained. The combination of these factors poses considerable challenges when designing diets for use in a dietary intervention trial.

This report aims to provide details of the methods, calculations and approaches used to achieve specific modifications in SFA and MUFA intakes, while leaving other dietary components unchanged, in a group of students living in a fully catered university hall of residence. The degree to which the original dietary targets were achieved in the final dietary intervention, and the factors that contributed to the variance from the target intakes, was also evaluated. This study has revealed important theoretical issues that need to be introduced in the planning and design of experimentally modified diets, as well as practical problems of implementation. Although the detail was specific to the present dietary intervention trial, the principles may be applied to the design of experimental diets in other settings, including free-living populations $^{16,17}$.

\section{Study design and dietary targets}

The dietary intervention study had a randomised, single blind parallel design, and was carried out in a fully catered hall of residence at the University of Reading. Fifty-one students (26 males and 25 females) participated in the study. All subjects consumed a reference (SFA) diet for 8 weeks. They were then randomly assigned to receive either a moderate MUFA (MM) diet or high MUFA (HM) diet for a further 16 weeks. The effects of these diets on a range of fasting and postprandial atherogenic and thrombogenic risk indicators were assessed, and preliminary results have been presented in the form of abstracts $^{18,19}$.

The students were provided with three meals a day, including both a cooked lunch and dinner, with the exception of evening meals at the weekend. All meals were prepared and served from a single kitchen. It was intended that changes in the fatty acid composition of the students' diet would be largely implemented by altering the fats used for food preparation, in spreading and in fats added directly to food, thereby allowing the use of the kitchen's usual menus and recipes. This was important, as a major aim of the study was to assess the feasibility and effects of increasing MUFA intake using typical UK foods.

The target fatty acid compositions of the three experimental diets are shown in Table 1 . The reference diet aimed to reflect the composition of the typical UK $\operatorname{diet}^{20}$ and its purpose was to standardise the fatty acid intake of all subjects prior to the start of the MUFA diet period. This was important owing to the multi-ethnic nature of the student population. The target total fat content of the reference diet was 37\% of dietary energy, since prior assessment of the students' habitual energy and nutrient intake (described below) indicated that this was close to the mean intake for this group. The moderate and high MUFA diets aimed to substitute $16 \%$ and 33\% of SFA, respectively, with MUFA. An important design criterion was that the diets should differ only in the proportions of SFA and MUFA, while maintaining constant intakes of energy, PUFA and trans-fatty acids. It was also necessary for the ratio of palmitic to stearic acid, and of $n-6$ to $n-3$ polyunsaturated fatty acids, to be kept constant between the diets, due to possible differences in plasma lipid responses to individual fatty acids within the same fatty acid class ${ }^{6}$.

Table 1 Target fatty acid compositions of the reference diet, moderate MUFA diet and high MUFA diet, compared with the students' habitual hall diet

\begin{tabular}{|c|c|c|c|c|c|}
\hline & \multicolumn{2}{|c|}{$\begin{array}{l}\text { Habitual hall diet } \\
(n=19) \\
\text { (measured) }\end{array}$} & \multicolumn{3}{|c|}{ Intervention diet targets (calculated) } \\
\hline & Mean & SD & Reference (SFA) diet & Moderate MUFA diet & High MUFA diet \\
\hline \multicolumn{6}{|l|}{$\%$ Energy as } \\
\hline Total fat & 36.0 & 3.5 & 36.8 & 36.8 & 36.8 \\
\hline SFA & 12.3 & 2.1 & 15.6 & 13.1 & 10.5 \\
\hline cis-MUFA & 12.9 & 1.4 & 11.8 & 14.6 & 17.2 \\
\hline cis-PUFA & 4.9 & 0.8 & 5.8 & 5.8 & 5.8 \\
\hline trans-FA & 1.9 & 0.4 & 0.7 & 0.7 & \\
\hline Ratio $16: 0$ to $18: 0$ & 2.2 & 0.4 & 2.2 & 2.2 & 2.2 \\
\hline Ratio $n-6$ to $n-3$ PUFA & 5.7 & 0.8 & 6.9 & 6.9 & 6.9 \\
\hline
\end{tabular}

SD - standard deviation; SFA - saturated fatty acids; MUFA - monounsaturated fatty acids; PUFA - polyunsaturated fatty acids; FA - fatty acids; 16:0 palmitic acid; 18:0 - stearic acid. 


\section{Methods}

\section{Dietary analysis and calculations}

All dietary analysis in this study was performed using the Foodbase Nutrition Database ${ }^{21}$, which has a number of features that make it suitable for analysing and designing diets of different fatty acid composition. In particular, the 'Fat Finder' facility allows individual foods (Fat Finder Host foods) to be entered along with the fat source used as an ingredient or in the cooking method (Fat Finder Source foods). This enables the amounts and types of added fat from different fat sources in the diet to be quantified. Added dietary fats such those used for spreading or cooking may be said to be 'exchangeable fat sources' as they can be substituted for alternatives. This is in contrast with 'intrinsic fats' which include those within dairy products, meats and 'ready-made' foods. Once a diet record for an individual, or a series of diet records from a group of people, have been entered into Foodbase, the record(s) can be edited to substitute the exchangeable fats with theoretical alternatives (e.g. butter can be 'replaced' with margarine). This allows theoretical modification of the fatty acid composition of the diet and enables identification of the fatty acid compositions of fats needed to achieve the dietary fatty acid target intakes.

\section{Assessment of habitual diet}

Prior to the start of the main intervention trial, the habitual diet of a group of students in this hall of residence was assessed. Nineteen students (11 males and eight females) completed a 7-day diet diary, which took the form of a preprinted booklet listing the individual food items on the hall menu at each meal. Students were asked to tick their choices and average portion sizes were determined by the investigators, who weighed at least three typical portions of each food. Other food and drink items consumed outside the hall dining room were also recorded, with portion sizes estimated using household measures. The weights of these foods were later estimated using tables of standard portion sizes ${ }^{22}$ and food labels where available.

\section{Customisation of Foodbase}

Details of the recipes, cooking methods and ingredients used were obtained from the catering staff. The fatty acid composition of all cooking fats and spreads used in the hall were analysed (Reading Scientific Services Ltd (RSSL), University of Reading) and their fatty acid compositions added to Foodbase as new food records. Recipes for meals prepared in the hall kitchen were entered into Foodbase and used to create new food records (Fat Finder Host foods). It was found that approximately $50 \%$ of the foods served were either partly or fully prepared prior to delivery, and many were subsequently oven-baked or deep-fried. Information about the composition of these pre-prepared foods, where available, was obtained from the suppliers or manufacturers. This usually took the form of a list of ingredients with the energy and macronutrient composition of the product. Details of fatty acid classes were available for some, but not all foods. Using the manufacturer's list of ingredients, a recipe was created in Foodbase and the proportions of the different ingredients adjusted until the composition of the food matched closely that provided. The weight loss of oven-baked foods was determined by the mean difference in weight of at least five items before and after baking, or by using published information for similar foods ${ }^{23}$. The fat uptake of deepfried foods was determined by direct analysis of their total fat content before and after frying using the Soxflo extraction technique ${ }^{24}$, and this was incorporated into Foodbase as the 'Fat Finder Value' for each food.

\section{Identification of exchangeable fat sources}

The 19 dietary records were analysed using this modified Foodbase database, and the daily intake of each exchangeable fat source was calculated. These data revealed that the target fatty acid compositions of the intervention diets could not be achieved by substitution of the exchangeable fats alone, since the exchangeable fat sources accounted for less than 30\% of total daily fat intake (see Results section). It was recognised that substitution of snack foods with specially manufactured alternatives, and the provision of evening meals at the weekend, would be required to increase the students' intake of experimental fats during the study. It was also hoped that they could be encouraged to increase their intake of spreads (e.g. on toast and by providing them with crackers). As the students had only limited facilities for food preparation and storage, they were provided with a variety of easily prepared low-fat tinned or packet foods to which they could add the experimental fat (as spread) during cooking.

The 19 diet records were edited on Foodbase to remove approximately three-quarters of the recorded snack foods (biscuits, cakes and crisps) consumed every day, and also meals prepared or bought by the students at the weekend. These were 'replaced' with biscuits providing $20 \mathrm{~g}$ margarine per day (to represent snacks), two low-fat ready meals plus $40 \mathrm{~g}$ margarine per week (to represent weekend evening meals) and an additional $10 \mathrm{~g}$ spread per day. A commercially available margarine ( $80 \%$ fat) was used as the fat source for these additional foods. These strategies increased the amount of potentially exchangeable fat by $24 \mathrm{~g} \mathrm{day}^{-1}$ on weekdays and $40 \mathrm{~g} \mathrm{day}^{-1}$ at the weekend.

\section{Determining fat sources required for the intervention diets}

The 19 edited diet diaries were used as the basis for calculations that would allow identification of the fatty acid compositions of the experimental fat sources to be used for each intervention diet. The process of designing the three diets was an iterative one, and required up to 10 different combinations of theoretical fat sources to be tried 
for each diet. For practical reasons (see Discussion), the moderate and high MUFA diets were designed to use the same fat sources in the hall kitchen, with the difference in composition of the two diets achieved by the use of different spreads and snack foods for each group. Although the calculations showed it would be possible to use commercially available fats for cooking, it became apparent that, to meet all required targets, margarines of specific fatty acid composition would need to be formulated (see Results section).

\section{Manufacture of experimental margarines and additional foods}

Having determined the target composition of experimental margarines for the three diets, suitable fat blends were identified and the margarines were manufactured (Van den Bergh Oils, Purfleet, UK). They were packaged in $250 \mathrm{~g}$ tubs, which were provided to the students for spreading and adding to other foods, and also in $2 \mathrm{~kg}$ catering packs for use in the hall kitchen. The margarines were used in the preparation of a range of snack foods for each diet, including three varieties of biscuit (manufactured by United Biscuits Ltd, High Wycombe, UK), and a number of different cake products (baked at the University of Reading). Two flavours of crisps were manufactured, using different oils for each of the experimental diets (United Biscuits Ltd, High Wycombe, UK). The Foodbase database was modified to include the experimental margarines (analysed by RSSL, University of Reading) and the manufactured snack foods (analysed by United Biscuits). Recipes for the baked products were also added to Foodbase.

\section{Implementation of the diets}

In order for the margarine and additional foods to be incorporated into the students' diet, a flexible lipid unit system was devised whereby one unit was equivalent to $8.5 \mathrm{~g}$ of exchangeable fat (equivalent to $10.6 \mathrm{~g}$ of experimental margarine). Portions of the margarine or snack foods were assigned either one or two lipid units, depending on the amount of experimental fat they contained (Table 2). The students were required to eat the majority of their meals in hall, and to consume an average of four lipid units a day on weekdays and six units a day at the weekend. The additional units at the weekend

Table 2 Lipid unit contents of manufactured intervention foods

\begin{tabular}{llc}
\hline Food & Portion & Lipid units \\
\hline Margarine & $10.6 \mathrm{~g}$ & 1 \\
Digestive biscuits & 3 & 1 \\
Boaster biscuits & 2 & 1 \\
Hobnob biscuits & 3 & 1 \\
Crisps & $25.5 \mathrm{~g}$ bag & 1 \\
Muffins (six varieties) & 1 piece & 1 \\
Cakes - flapjack, shortbread, & 1 piece & 2 \\
$\quad$ lemon sponge, etc. & & \\
\hline
\end{tabular}

were to compensate for the fact that they were not provided with an evening meal in hall on these days. Each student had their own tub of margarine available in the dining room at meal times, and was also provided with tubs for use at other times. The investigators met with each student at least once a fortnight during the study, and provided them with a supply of snack foods and low-fat meals. All margarines and snack foods were colour-coded, and the students were not told which diet they were on.

Students were asked to complete two 4-day diet diaries during the study, one each during the reference and MUFA diet phases. To provide an objective measure of dietary compliance, plasma phospholipid and cholesteryl ester fatty acid compositions were measured at the beginning and end of the MUFA diet period, using the method of capillary gas chromatography as described previously ${ }^{25}$. Specific fatty acids were identified by reference to methyl ester standards and peak areas were corrected for individual response factors. Results were expressed as percentage composition by weight $(\mathrm{g} / 100 \mathrm{~g}$ total fatty acids).

\section{Statistical analysis}

Data are presented in the tables as the mean and standard deviation (SD). Statistical analysis was performed using SPSS for Windows (Version 9.0). Data were checked for normality and log transformation carried out where necessary. Comparisons between the variables measured on the reference and MUFA diets were made within groups using paired analysis, and comparisons between the moderate MUFA and high MUFA groups were made using independent $t$-tests. A value of $P<0.05$ was considered to be statistically significant.

\section{Results}

\section{Design of intervention diets}

The mean energy, total fat and fatty acid intakes of the group of students consuming the habitual hall diet $(n=19)$ are shown in Table 1 . The habitual diet differed in a number of respects from the typical UK diet, being lower in total fat and SFA and higher in MUFA. The mean reported total fat intake for the group was 102.3 (SD 29.6) $\mathrm{g} \mathrm{day}^{-1}$, which represented 36.0\% (SD 3.5\%) of dietary energy.

The exchangeable fat sources provided by foods consumed in the hall dining room included fats used for deep and shallow frying, preparation of soups and sauces, baking and spreading (two types). The composition and mean daily intake of each of these fats are shown in Table 3. In addition, some students also used their own spreads and cooking oils for the preparation of additional food, e.g. at weekends (not shown in table). The mean daily intake of total exchangeable fat was 30.9 (SD 10.5) gday $^{-1}$. This represented 26.9 (SD 11.7) g day $^{-1}$ from food provided to the students in hall (Table 3), and an 
Table 3 Composition and mean daily intakes of exchangeable fat sources in the students' habitual diet

\begin{tabular}{|c|c|c|c|c|c|c|}
\hline & \multicolumn{6}{|c|}{ FA composition $(\mathrm{g} / 100 \mathrm{~g})$} \\
\hline & \multicolumn{3}{|c|}{ Oils } & \multicolumn{3}{|c|}{ Margarine/spread } \\
\hline & $\begin{array}{l}\text { Deep } \\
\text { frying }\end{array}$ & $\begin{array}{l}\text { Shallow } \\
\text { frying }\end{array}$ & Sauces & $\begin{array}{l}\text { Baking } \\
\text { margarine }\end{array}$ & $\begin{array}{l}\text { Spread } \\
1 \text { (butter) }\end{array}$ & $\begin{array}{c}\text { Spread } \\
2 \text { (Flora) }\end{array}$ \\
\hline Total FA & 95.5 & 95.9 & 93.9 & 77.1 & 78.3 & 67.2 \\
\hline Total SFA & 13.8 & 7.2 & 13.1 & 21.6 & 52.0 & 14.5 \\
\hline $16: 0$ & 5.5 & 4.6 & 5.4 & 9.4 & 21.1 & 8.7 \\
\hline $18: 0$ & 7.1 & 1.6 & 6.6 & 5.4 & 9.5 & 2.3 \\
\hline Total cis-MUFA & 52.7 & 60.0 & 58.2 & 36.2 & 19.2 & 21.0 \\
\hline $18: 1$ & 47.0 & 59.3 & 54.7 & 35.5 & 16.1 & 19.8 \\
\hline Total cis-PUFA & 7.7 & 28.3 & 6.2 & 18.9 & 1.1 & 31.3 \\
\hline $18: 2 n-6$ & 6.8 & 19.8 & 5.3 & 13.4 & 0.7 & 29.8 \\
\hline $18: 3 n-3$ & 0.7 & 7.7 & 0.8 & 5.4 & 0.4 & 1.5 \\
\hline Total trans-FA & 21.3 & 0.4 & 16.4 & 0.4 & 4.8 & 0.4 \\
\hline Fat intake $\left(\mathrm{g} \mathrm{day}^{-1}\right)$, mean (SD) & $10.8(3.8)$ & $2.1(1.1)$ & $1.6(0.7)$ & $5.6(3.4)$ & $4.8(5.9)$ & $2.1(4.4)$ \\
\hline
\end{tabular}

FA - fatty acids; SFA - saturated fatty acids; MUFA - monounsaturated fatty acids; PUFA - polyunsaturated fatty acids; 16:0 palmitic acid; 18:0 - stearic acid; 18:1 - oleic acid; 18:2n - 6 - linoleic acid; $18: 3 n-3-\alpha$-linolenic acid; SD - standard deviation.

additional 4.0 (SD 5.6) $\mathrm{g} \mathrm{day}^{-1}$ from other fat sources used by the students themselves. The greatest exchangeable fat source was that contributed by deep-fried foods (10.8 (SD 3.8) $\mathrm{g} \mathrm{day}^{-1}$ ) followed by spreads (a total of 6.9 (SD 6.5) $\mathrm{g} \mathrm{day}^{-1}$ ). As the exchangeable fat sources accounted for only one-third of total fat intake, alterations in the MUFA and SFA contents of this relatively small amount of exchangeable fat could not enable the target fatty acid compositions of the three diets to be achieved by this means alone.

The addition of modified snacks and spreads to the diet records allowed the mean amount of potentially exchangeable fat in the diet to be increased from approximately 31 to $55 \mathrm{~g} \mathrm{day}^{-1}$. Seven main uses of exchangeable fat were then available to the investigators, including four in the hall kitchen (deep frying, shallow frying, sauces and baking) and three via foods provided directly to individual students (spreads, fats added to low-fat meals and snack foods).

The fats identified for the three experimental diets are listed with their uses in Table 4, and the composition of each of these fats is presented in Table 5. The cooking fats selected for the reference and MUFA diets were suitable for both deep and shallow frying. These were a solid vegetable lard (41\% SFA, 42\% MUFA and 11\% PUFA) and a vegetable oil (vegetable oil 1) consisting largely of rapeseed oil (7\% SFA, 60\% MUFA and 29\% PUFA). Although both were relatively low in trans-fatty acids $(<1 \%)$, the vegetable lard contained almost twice as much as the vegetable oil ( $0.7 \%$ compared with $0.4 \%)$. In order to prevent this resulting in a difference in the trans-fatty acid composition of the SFA and MUFA diets, a flavoured hydrogenated vegetable oil was used for the preparation of sauces during the MUFA diet phase of the study. This oil (vegetable oil 2) contained $16.4 \%$ trans-fatty acids, but as it was used in much smaller quantities than the fats used for frying, it made only a small contribution to trans-fatty acid intake.

The target fatty acid compositions of a low, moderate and high MUFA margarine were defined, and suitable oil blends were identified (Table 5). The low MUFA margarine (43\% SFA-rich hard stock margarine, 33\% rapeseed oil and 23\% sunflower oil), moderate MUFA margarine (40\% SFA-rich hard stock margarine, $10 \%$ rapeseed oil, $22 \%$

Table 4 Fat sources used in the intervention diets

\begin{tabular}{|c|c|c|c|}
\hline & \multicolumn{3}{|c|}{ Intervention diet } \\
\hline & Reference (SFA) & Moderate MUFA & High MUFA \\
\hline \multicolumn{4}{|l|}{ Used in hall kitchen } \\
\hline Deep frying & Vegetable lard & \multicolumn{2}{|c|}{ Vegetable oil 1} \\
\hline Shallow frying & Vegetable lard & \multicolumn{2}{|c|}{ Vegetable oil 1} \\
\hline Sauces & Low MUFA margarine* & \multicolumn{2}{|c|}{ Vegetable oil 2} \\
\hline Baking/puddings & Low MUFA margarine* & \multicolumn{2}{|c|}{ High MÜFA margarine* } \\
\hline \multicolumn{4}{|l|}{ Used by students } \\
\hline Spreading & Low MUFA margarine* & Moderate MUFA margarine* & High MUFA margarine* \\
\hline Added to low fat meals & Low MUFA margarine* & Moderate MUFA margarine* & High MUFA margarine* \\
\hline Snack foods & Low MUFA margarine* & Moderate MUFA margarine* & High MUFA margarine* \\
\hline
\end{tabular}

SFA - saturated fatty acids; MUFA - monounsaturated fatty acids.

* These were prepared according to the desired fatty acid profile of each of the diets. 
Table 5 Composition of fat sources used for intervention diets

\begin{tabular}{|c|c|c|c|c|c|c|c|c|c|}
\hline & \multicolumn{9}{|c|}{ FA composition $(\mathrm{g} / 100 \mathrm{~g})$} \\
\hline & \multirow{2}{*}{\multicolumn{3}{|c|}{ Commercially available cooking fats }} & \multicolumn{6}{|c|}{ Experimental margarines } \\
\hline & & & & \multicolumn{2}{|c|}{$\begin{array}{l}\text { Low MUFA } \\
\text { margarine }\end{array}$} & \multicolumn{2}{|c|}{$\begin{array}{c}\text { Moderate } \\
\text { MUFA margarine }\end{array}$} & \multicolumn{2}{|c|}{$\begin{array}{l}\text { High MUFA } \\
\text { margarine }\end{array}$} \\
\hline & $\begin{array}{l}\text { Vegetable lard } \\
\text { (SFA diet) }\end{array}$ & $\begin{array}{l}\text { Vegetable oil } 1 \\
\text { (MUFA diets) }\end{array}$ & $\begin{array}{l}\text { Vegetable oil } 2 \\
\text { (MUFA diets) }\end{array}$ & Target & Analysed & Target & Analysed & Target & Analysed \\
\hline Total FA & 94.1 & 95.9 & 93.9 & 78.6 & 76.0 & 76.0 & 76.0 & 76.0 & 76.0 \\
\hline Total SFA & 41.2 & 7.2 & 13.1 & 36.1 & 32.4 & 32.5 & 30.3 & 10.0 & 12.5 \\
\hline $16: 0$ & 24.8 & 4.6 & 5.4 & 24.6 & 20.4 & 21.7 & 19.2 & 6.7 & 6.8 \\
\hline $18: 0$ & 15.2 & 1.6 & 6.6 & 11.5 & 2.7 & 10.8 & 3.1 & 3.3 & 2.8 \\
\hline Total cis-MUFA & 41.0 & 60.0 & 58.2 & 22.5 & 23.2 & 32.5 & 29.6 & 55.0 & 46.5 \\
\hline $18: 1$ & 38.1 & 59.3 & 54.7 & 22.5 & 22.7 & 32.5 & 29.3 & 55.0 & 46.1 \\
\hline Total cis-PUFA & 11.2 & 28.3 & 6.2 & 20.0 & 20.2 & 15.0 & 15.9 & 15.0 & 16.9 \\
\hline $18: 2 n-6$ & 9.8 & 19.8 & 5.3 & 17.3 & 17.8 & 14.2 & 15.2 & 14.2 & 16.1 \\
\hline $18: 3 n-3$ & 1.0 & 7.7 & 0.8 & 2.7 & 2.4 & 0.8 & 0.7 & 0.8 & 0.8 \\
\hline Total trans-FA & 0.7 & 0.4 & 16.4 & 0 & 0.2 & 0 & 0.2 & 0 & 0.2 \\
\hline
\end{tabular}

FA - fatty acids; SFA - saturated fatty acids; MUFA - monounsaturated fatty acids; PUFA - polyunsaturated fatty acids; 16:0 - palmitic acid; 18:0 stearic acid; $18: 1$ - oleic acid; $18: 2 n-6$ - linoleic acid; 18:3n-3- $\alpha$-linolenic acid.

sunflower oil, 28\% high oleic sunflower oil) and high MUFA margarine (9\% SFA-rich hard stock margarine, 10\% rapeseed oil, $20 \%$ sunflower oil, $61 \%$ high oleic sunflower oil) were manufactured in single batches that provided sufficient quantities for the entire study. The vitamin $\mathrm{E}$ composition of the low, moderate and high MUFA

Table 6 Reported energy, total fat and fatty acid intakes of the moderate MUFA (MM) group, high MUFA (HM) group and the two groups combined on the reference and experimental (MUFA) diets

\begin{tabular}{|c|c|c|c|c|c|c|}
\hline & \multicolumn{2}{|c|}{ MM group } & \multicolumn{2}{|c|}{ HM group } & \multicolumn{2}{|c|}{$\begin{array}{c}\text { Combined } \\
\text { groups }\end{array}$} \\
\hline & Mean & SD & Mean & SD & Mean & SD \\
\hline \multicolumn{7}{|l|}{ Reference diet } \\
\hline $\begin{array}{l}\text { Energy (MJ) } \\
\% \text { Energy as }\end{array}$ & 11.0 & 2.3 & 10.8 & 1.7 & 10.9 & 2.0 \\
\hline Total fat & 39.8 & 4.6 & 37.7 & 4.6 & 38.7 & 4.7 \\
\hline SFA & 15.4 & 2.1 & 14.5 & 2.0 & 14.9 & 2.1 \\
\hline cis-MUFA & 12.5 & 1.8 & 11.9 & 1.7 & 12.2 & 1.7 \\
\hline cis-PUFA & 7.3 & 1.1 & $6.4 \dagger$ & 1.2 & 6.8 & 1.2 \\
\hline trans-FA & 1.0 & 0.4 & 0.9 & 0.3 & 0.9 & 0.3 \\
\hline Ratio $16: 0$ to 18 : & 3.0 & 0.6 & 2.9 & 0.5 & 2.9 & 0.6 \\
\hline Ratio $n-6$ to $n-3$ PUFA & 7.1 & 0.6 & 7.0 & 1.2 & 7.0 & 0.9 \\
\hline \multicolumn{7}{|l|}{ Experimental diet } \\
\hline Energy (MJ) & 10.7 & 2.2 & 10.5 & 1.6 & 10.6 & 1.9 \\
\hline \multicolumn{7}{|l|}{$\%$ Energy as } \\
\hline Total fat & 39.7 & 4.2 & 37.1 & 4.2 & 38.4 & 4.3 \\
\hline SFA & $12.1^{\star \star}$ & 1.7 & $9.9^{* \star} \dagger \dagger$ & 1.9 & $11.0^{\star *}$ & 2.1 \\
\hline cis-MUFA & $15.3^{\star *}$ & 2.1 & $16.3^{\star \star}$ & 2.4 & $15.8^{\star \star}$ & 2.3 \\
\hline cis-PUFA & 7.2 & 1.1 & $6.5 \dagger$ & 0.8 & 6.9 & 1.0 \\
\hline & 0.8 & 0.2 & 0.8 & 0.2 & 0.8 & 0.2 \\
\hline 0 to $18: 0$ & 2.9 & 0.4 & $2.4^{*} \dagger$ & 0.3 & $2.7^{*}$ & 0.4 \\
\hline Ratio $n-6$ to $n-3$ PUFA & 6.3 & 1.5 & 7.3 & 1.8 & 6.8 & 1.7 \\
\hline
\end{tabular}

SD - standard deviation; SFA, saturated fatty acids; MUFA, monounsaturated fatty acids; PUFA, polyunsaturated fatty acids; FA - fatty acids; 16:0 - palmitic acid; 18:0 - stearic acid.

MUFA diet significantly different from reference diet: *, $P<0.05$; **, $P<0.001$

$\mathrm{HM}$ group significantly different from $\mathrm{MM}$ group: $\dagger, \quad P<0.05$ ††, $P<0.001$. margarine was $27.7 \mathrm{mg} / 100 \mathrm{~g}, \quad 25.5 \mathrm{mg} / 100 \mathrm{~g}$ and $26.7 \mathrm{mg} / 100 \mathrm{~g}$, respectively.

On analysis, a number of differences between the target compositions and the analysed compositions of the manufactured margarines were subsequently found (Table 5). In particular, the levels of SFA in the low MUFA margarine and MUFA in the high MUFA margarine were both lower than the desired levels. The other major deviation from the target compositions of the experimental margarines was in the ratio of palmitic to stearic acid, which had been planned to be the same for each of the three margarines, but in practice was considerably higher in the low and moderate MUFA margarines than in the high MUFA margarine (ratios of 7.6, 6.2 and 2.4, respectively). The actual $n-6$ to $n-3$ PUFA ratios of the low, moderate and high MUFA margarines were 7.4, 21.8 and 20.1, respectively, and were all slightly higher than the target ratios of $6.4,17.8$ and 17.8 .

\section{Achievement of dietary fatty acid targets}

The reported energy, total fat and fatty acid intakes of the students on the reference diet and their respective MUFA diets are shown in Table 6. Data are presented for 42 individuals on the reference diet and 43 on the MUFA diets. There was no difference in the reported energy intakes between the moderate and high MUFA groups on either the reference or MUFA diet (Table 6). During both dietary phases, there was a tendency for the MM group to consume a greater proportion of dietary energy as fat and less as carbohydrate (data not included in table) than did the HM group, although these differences did not reach statistical significance during either dietary phase. The between-group differences in reported fat intake were largely attributable to differences between the males of the two groups. The MM males reported total fat intakes of $41.9 \%$ (SD 3.2\%) and $41.4 \%$ (SD 4.3\%) for the reference 
and MUFA diet phases, respectively, compared with reported intakes by the HM males of $38.7 \%$ (SD 3.2\%) and $37.0 \%$ (SD 5.5\%) (data for males only not shown in table). This difference between the groups was statistically significant only during the reference diet phase $(P<0.05)$, due to a greater range of reported total fat intakes in the HM males on the MUFA diet. The reported energy and fat intakes did not differ within either group, or when the two groups were combined, as the students went from the reference diet to their respective MUFA diets.

The dietary intervention largely achieved the target fatty acid composition of the reference diet with the group as a whole reporting intakes of $14.9 \%$ SFA and $12.2 \%$ cis-MUFA (Table 6), compared with the targets of $15.6 \%$ SFA and $11.8 \%$ cis-MUFA. The reported contribution of all fatty acid classes to total energy intake was lower in the HM group than in the MM group due to their lower total fat intake, but this difference was significant only for cis-PUFA intake $(P<0.05)$.

Both the moderate and the high MUFA diet achieved significant increases in cis-MUFA intake $(P<0.001)$ and reductions in SFA intake $(P<0.001)$. The high MUFA diet intervention resulted in a mean difference of $6.4 \%$ (SD $2.6 \%$ ) in the percentage energy from SFA and MUFA, which was similar to the target difference of $6.7 \%$. A mean difference of $3.1 \%$ (SD 1.9\%) was achieved on the moderate MUFA diet; this was greater than the target difference of $1.5 \%$ energy, and was the result of both a lower mean SFA intake and a higher mean MUFA intake than anticipated from the dietary calculations. As a result of the differences in absolute total fat intake of the two groups, and the fact that the MM group exceeded the target intake for MUFA while the HM group did not reach the target, the mean absolute MUFA intakes ( $\mathrm{g}$ ) of the two groups were not actually different (data not shown).

Despite the reported differences between the groups in the percentage energy consumed as total fat and PUFA on both diets, there were no within-group differences in the intake of cis-PUFA or trans-fatty acids as the students went from the reference diet to their respective MUFA diets.

The reported ratios of palmitic to stearic acid intake, and $n-6$ to $n-3$ PUFA intake, are shown in Table 6 . The group as a whole showed a small but significant reduction in the palmitic to stearic acid ratio $(P<0.05)$ during the MUFA diet compared to the reference diet, which was due to a reduction in the HM group $(P<0.05)$ but not the MM group. There were no between-group differences in the palmitic to stearic acid ratio on the reference diet, but during the MUFA diet phase the ratio was lower in the HM group than the MM group $(P<0.05)$. There were no significant differences in the ratio of $n-6$ to $n-3$ PUFA intake between the groups during either dietary phase, or within groups as they went from the reference to MUFA diets.

The changes in plasma phospholipid fatty acids and
Table 7 Fatty acid composition of plasma phospholipids on the reference and MUFA diets for MM, HM and combined groups

\begin{tabular}{|c|c|c|c|c|c|c|}
\hline & \multicolumn{2}{|c|}{ MM group } & \multicolumn{2}{|c|}{ HM group } & \multicolumn{2}{|c|}{$\begin{array}{l}\text { Combined } \\
\text { groups }\end{array}$} \\
\hline & Mean & SD & Mean & SD & Mean & SD \\
\hline \multicolumn{7}{|c|}{ Reference diet ( $\mathrm{g} / 100 \mathrm{~g}$ total $\mathrm{FA}$ ) } \\
\hline SFA & 44.74 & 0.93 & 45.40 & 3.50 & 45.44 & 3.42 \\
\hline MUFA & 11.63 & 1.12 & 11.76 & 1.62 & 11.59 & 1.39 \\
\hline PUFA & 43.63 & 1.55 & 42.84 & 4.40 & 42.96 & 4.04 \\
\hline \multicolumn{7}{|c|}{ Experimental diets ( $\mathrm{g} / 100 \mathrm{~g}$ total $\mathrm{FA})$} \\
\hline SFA & 44.40 & 1.45 & 43.95 & 1.88 & $44.39^{\star}$ & 2.78 \\
\hline MUFA & 11.71 & 1.35 & 12.69 & 1.60 & $12.24^{\star *}$ & 1.47 \\
\hline PUFA & 43.89 & 1.44 & 43.36 & 2.41 & 43.36 & 3.14 \\
\hline
\end{tabular}

MM - moderate MUFA; HM - high MUFA; SD - standard deviation; FA fatty acids; SFA - saturated fatty acids; MUFA - monounsaturated fatty acids; PUFA - polyunsaturated fatty acids.

Significant change from end of reference diet: ${ }^{*}, P<0.05 ;{ }^{\star \star}, P<0.01$.

cholesteryl ester fatty acids from the end of the reference diet to the end of the MUFA diet phase are shown in Table 7. The group as whole showed a significant reduction in plasma phospholipid SFA composition $(P<0.05)$, with a corresponding increase in MUFA $(P<0.01)$. Similar changes occurred in the SFA and MUFA composition of cholesteryl esters, although the changes were not statistically significant. When the MM and HM groups were considered separately, the only significant change was in the reduction in cholesteryl ester SFA in the HM group $(P<0.01)$. However, within both the phospholipid and the cholesteryl ester fractions, there was a tendency for the changes to be greater in the HM group than the MM group, indicating that there were dietary differences between the two groups. There were no significant changes in the PUFA composition of plasma phospholipids or cholesteryl esters in response to the dietary intervention.

\section{Discussion}

The main purpose of this report was to describe the methods employed in the design and implementation of experimental diets of differing SFA and MUFA composition for use in a dietary intervention trial in a university hall of residence. An additional aim was to assess the outcome of the dietary intervention in terms of the achievement of the dietary targets.

The study required the design of three experimental diets that differed only in their SFA and MUFA compositions, while keeping all other dietary components constant between the diets. These targets were largely achieved, with significant reductions in SFA intake and increases in MUFA intake in both dietary groups, although the difference in the final MUFA intake of the two groups was not as great as had been predicted. The reported changes in SFA and MUFA intake were supported by the changes in the plasma biomarkers. Although there were differences in the total fat intake of the MM and HM groups 
that had not previously been anticipated, there were no within-group differences in the intake of fat or the major fatty acid classes as the students went from the reference diet to their respective MUFA diets.

Controlled dietary intervention studies involving the manipulation of dietary fatty acids have previously been carried out in residential settings in which subjects had all of their meals prepared in a central kitchen ${ }^{7,26}$. Because subjects had only very limited access to other foods, compliance was high. It has also been shown to be feasible to increase the MUFA content of a diet consisting of typical UK foods in free-living subjects by providing them with experimental fats for cooking and spreading, and also with manufactured ready-to-eat meals and snack foods ${ }^{16,17}$. This approach requires a greater level of subject commitment during the intervention trial, but may reflect ways of implementing dietary change that are more applicable in a wider setting.

In the present study, a combination of these approaches was used. By performing the intervention trial in a hall of residence, significant changes in the fatty acid composition of the diet could be achieved by altering the fats used in food preparation and spreading, with minimal inconvenience to the subjects themselves. Owing to the high proportion of pre-prepared foods that were habitually served in the hall dining room (the fatty acid composition of which could not be altered), the additional use of manufactured snack foods and margarines was required to allow greater modifications to be made to the fatty acid composition of the diet. Although the practical issues associated with implementing the diets were determined by the setting of the study, the process involved in their design has wider application, and may be modified for use in other settings, particularly in free-living populations where pre-prepared foods are also commonly consumed.

In order to design any experimental diet, a suitable nutrient database is required, which should meet certain criteria $^{27}$. Where specific alterations are to be made to the fatty acid composition of the diet, the database should contain individual fatty acid compositions (not just fatty acid classes) of a significant number of foods, especially those foods typically consumed by the study population. It should also include up-to-date analysis of fats, oils and spreads. The dietary analysis program should also allow the addition of new foods to the database, and enable existing foods to be updated if more recent information about their fatty acid composition becomes available (e.g. from manufacturers or following laboratory analysis). The Foodbase Database ${ }^{21}$ that was used to design the diets in the present study met these criteria, and had the additional advantage of its 'Fat Finder Facility', which allowed the amount of fat incorporated into many foods, either as an ingredient or as part of the cooking method, to be calculated automatically and listed separately. This facilitated the subsequent theoretical substitution of one type of exchangeable fat with another when designing the intervention diets.

The type of dietary intervention most likely to be acceptable to subjects in long-term studies is one in which changes are made to the fatty acid composition of the diet by altering the fat sources, while keeping the types of foods habitually consumed the same. An initial assessment of the diet of a group of students in this hall was therefore carried out to identify their dietary habits and to determine the types and quantities of exchangeable fat in their diet. These dietary records were also used as the basis for making the subsequent theoretical fat substitutions required to meet the target compositions of the diets. A more accurate assessment of dietary fatty acid intake could be made in this setting than is generally possible in freeliving individuals, because the investigators had detailed information about recipes, ingredients, fat sources and portion sizes, and the database could be modified to include these. In addition, there was less variability in the types of fats consumed than would occur in subjects living in their own homes and preparing their own food. When designing experimental diets for free-living individuals, where there will be greater variability between individuals, it may be more practical to define a common diet based on diet diaries completed by a group of subjects similar to the proposed study population, and to use this as a basis for making the subsequent fat substitutions ${ }^{16,17}$.

Assessment of the habitual diet revealed that the fatty acid targets of the intervention diets could not be reached by altering the exchangeable fat sources alone. This was partly because approximately 50\% of the food items served in the hall were pre-prepared manufactured foods. This reflects similar intakes of ready-made manufactured foods in free-living individuals ${ }^{17}$. In addition, as the students were typically provided with both a cooked lunch and dinner, their intake of spreads was lower than if they had consumed sandwiches more frequently. It has previously been shown that, in order to achieve MUFA intakes of greater than $20 \%$ of dietary energy, intervention of the whole diet (breakfast, lunch, dinner and snacks) is required ${ }^{17}$. This may be achieved by the provision of MUFA-enriched ready-to-eat meals, desserts, snacks and spreads produced in collaboration with food manufacturers. In the present study it was not practical to significantly alter the menus used in the hall to include fewer manufactured foods, or to produce MUFA-enriched alternatives. However, the amount of exchangeable fat was increased (from approximately 31 to $55 \mathrm{~g} \mathrm{day}^{-1}$ ) by the provision of experimental spreads and snack foods directly to the students.

Although the ideal fatty acid composition of the low, moderate and high MUFA margarines required to meet the dietary targets had been calculated, some compromise was required in selecting suitable fat blends for the manufacture of these margarines, because use of some fat sources led to an unacceptable feeling in the mouth 
and poor taste. These compromises resulted in some differences between the target composition and the final analysed composition of each of the margarines. This was an important cause of the lack of a significant difference in the MUFA intakes of the MM and HM groups, since the high MUFA margarine contained 15\% less MUFA than originally planned. The difference between the target and actual fatty acid compositions of the moderate and high MUFA margarines also contributed to small differences in the ratio of palmitic to stearic acid intake in the two diets, as the high MUFA margarine had a lower palmitic to stearic acid ratio than either the moderate or the low MUFA margarine. Due to problems of scheduling, it had not been possible to undertake analysis of the margarines prior to the study. Future studies should ensure complete analysis of all major fat sources prior to starting the study, although compromises may be inevitable due to problems of taste and performance of experimental fat sources.

The lipid unit system proved to be a flexible way of incorporating the experimental margarines into the diet, and most students found it relatively easy to consume the required quantity of units on a weekly basis. Students were offered a slightly different selection of snack foods at different times throughout the study to increase variety and interest. A wide selection of low-fat meals was also available for the students to use with their experimental margarine at weekends. Some students expressed negative feelings about the restricted choice of snack foods, particularly towards the end of the study, and so were encouraged to find alternative ways of consuming more spread. It should be noted that, in this study, the students were following experimental diets using specially prepared snacks for a total of 24 weeks, inevitably leading to product boredom. This emphasises the need for an adequate variety of acceptable intervention foods to be made available during long-term intervention studies, as well as regular monitoring of subjects. Although it was interesting to involve a commercial snack food manufacturer in the study, retrospectively it may have been preferable to bake more products locally, thereby enabling greater flexibility and choice.

Our experience in planning and carrying out this dietary intervention study indicates there are many factors which need to be considered to enable dietary targets to be achieved, while providing subjects with a diet that is both acceptable and practical to implement. In addition to the design of the experimental diets, sufficient consideration must be given to the quantities of experimental fats and intervention foods that will be required, their manufacture, analysis, packaging, delivery and storage arrangements, and distribution to the study participants. These factors inevitably affect the scope and cost of the study, as well as the morale of subjects and staff, which has a marked effect on subject adherence to the study protocol and dropout rate ${ }^{11}$. This study emphasises the need for both careful design of experimental diets, and sufficient consideration of the practical issues involved in their implementation, to ensure the successful outcome of a dietary intervention trial.

\section{Acknowledgements}

This study was funded by a grant from the Food Standards Agency. M.C.N. also received financial support from The Swedish Foundation for International Co-operation in Research and Higher Education. The authors acknowledge the contribution of Van den Bergh Foods Ltd, UK in providing the experimental fats, and United Biscuits Ltd, UK for manufacturing the snack foods. They also wish to thank the staff and students of St. George's Hall of Residence, University of Reading for their invaluable help and co-operation.

\section{References}

1 Department of Health. Nutritional Aspects of Cardiovascular Disease. Report on Health and Social Subjects No. 46. London: HMSO, 1994.

2 Pyorala K, De Backer G, Graham L. Prevention of coronary heart disease in clinical practice, recommendations of the Task Force of the European Society of Cardiology, European Atherosclerosis Society and European Society of Hypertension. Eur. Heart J. 1994; 15: 1300-31.

3 Mensink RP, Katan MB. Effect of dietary fatty acids on serum lipids and lipoproteins. A meta-analysis of 27 trials. Arteriosclerosis Thrombosis 1992; 12: 911-9.

4 Hegsted DM, Ausmann LM, Johnson JA, Dallal GE. Dietary fat and serum lipids: an evaluation of the experimental data. Am. J. Clin. Nutr. 1993; 57: 875-83.

5 Yu S, Derr J, Etherton TD, Kris-Etherton PM. Plasma cholesterol predictive equations demonstrate that stearic acid is neutral and monounsaturated fatty acids are hypocholesterolemic. Am. J. Clin. Nutr. 1995; 61: 1129-39.

6 Kris-Etherton PM, Yu S. Individual fatty acid effects on plasma lipids and lipoproteins: human studies. Am. J. Clin. Nutr. 1997; 65: 1628S-44S.

7 Berry EM, Eisenberg S, Haratz D, Friedlander Y, Norman Y, Kaufmann NA, et al. Effects of diets rich in monounsaturated fatty acids on plasma lipoproteins - the Jerusalem Nutrition Study; high MUFAs vs high PUFAs. Am. J. Clin. Nutr. 1991; 53: 899-907.

8 Aviram M, Eias K. Dietary olive oil reduces low-density lipoprotein uptake by macrophages and decreases the susceptibility of the lipoprotein to undergo lipid peroxidation. Ann. Nutr. Metab. 1993; 37: 75-84.

9 Roche HM, Zampelas A, Knapper JME, Webb D, Brooks C, Jackson KG, et al. The effect of chronic olive oil dietary intervention on acute postprandial triacylglycerol and factor VII metabolism. Am. J. Clin. Nutr. 1998; 68: 552-60.

10 Yaqoob P, Knapper JA, Webb DH, Williams CM, Newsholme EA, Calder PC. Effect of olive oil on immune function in middle aged men. Am. J. Clin. Nutr. 1998; 67: 129-35.

11 Kris-Etherton PM, Dietschy J. Design criteria for studies examining individual fatty acid effects on cardiovascular disease risk factors: human and animal studies. Am. J. Clin. Nutr. 1997; 65: 1590S-6S.

12 Becker N, Illingworth DR, Alaupovic P, Connor WE, Sundberg EE. Effects of saturated, monounsaturated and $n-6$ polyunsaturated fatty acids on plasma lipids, lipoproteins and apoproteins in humans. Am.J. Clin. Nutr. 1983; 37: $355-60$. 
13 Grundy SM. Comparison of monounsaturated fatty acids and carbohydrates for lowering plasma cholesterol. N. Engl. J. Med. 1986; 314: 745-8.

14 Bonanome A, Grundy SM. Effect of dietary stearic acid on plasma cholesterol and lipoprotein on levels. N. Engl.J. Med. 1988; 318: 1244-8.

15 Valsta LM, Jauhiainen M, Aro A, Katan MB, Mutanen M. Effects of a monounsaturated rapeseed oil and a polyunsaturated sunflower oil diet on lipoprotein levels in humans. Arteriosclerosis Thrombosis 1992; 12: 50-7.

16 Knapper JME, Tredger JA, Webb D, Culverwell C, Faulkner $\mathrm{W}$, Roche $\mathrm{H}$, et al. Substitution of dietary monounsaturated fatty acids for saturated fatty acids in a free-living population: a feasibility study. J. Hum. Nutr. Diet. 1996; 9: 273-82.

17 Williams CM, Francis-Knapper JA, Webb D, Brookes CA, Zampelas A, Tredger JA, et al. Cholesterol reduction using manufactured foods high in monounsaturated fatty acids, a randomised cross over study. Br.J. Nutr. 1999; 81: 439-46.

18 Smith RD, Kelly CNM, Silva KDRR, Nydahl MC, Williams CM. Effects of substituting dietary saturated fatty acids with monounsaturated fatty acids on blood lipids in young healthy volunteers. Proc. Nutr. Soc. 2001; 60: 45A.

19 Kelly CNM, Smith RD, Silva KDRR, Nydahl MC, Williams CM. Effects of substituting dietary saturated fatty acids with monounsaturated fatty acids on whole blood platelet aggregation in young healthy volunteers. Proc. Nutr. Soc. 2001; 60: 21A.
20 Ministry of Agriculture, Fisheries and Food. National Food Survey, 1995. London: HMSO, 1996.

21 The Institute of Brain Chemistry and Human Nutrition. Foodbase Nutrition Database and Software Package Version 1.3. London: The Institute of Brain Chemistry and Human Nutrition, 1993.

22 Ministry of Agriculture, Fisheries and Food. Food Portions Sizes. London: HMSO, 1993.

23 Holland B, Welch AA, Unwin ID, Buss DH, Paul AA, Southgate DAT. McCance \& Widdowson's The Composition of Foods, 5th ed. Cambridge, UK: The Royal Society of Chemistry, 1991.

24 Brown RH, Muller-Harvey I. Evaluation of the novel Soxflo technique for rapid extraction of crude fat in foods and animal feeds. J. AOAC 1999; 82: 1369-74.

25 Fielding BA, Callow J, Owen RM, Samra JS, Matthews DR, Frayn KN. Postprandial lipemia: the origin of an early peak studied by specific dietary fatty acid intake during sequential meals. Am.J. Clin. Nutr. 1996; 63: 36-41.

26 Mata P, Garrido JA, Ordovas JM, Blazquez E, Alvarez-Sala LA, Rubio MJ, et al. Effect of dietary monounsaturated fatty acids on plasma lipoproteins and apolipoproteins in women. Am. J. Clin. Nutr. 1992; 56: 77-83.

27 Buzzard IM, Price KS, Warren RA. Considerations for selecting nutrient-calculation software: evaluation of the nutrient database. Am. J. Clin. Nutr. 1991; 54: 7-9. 